\title{
ASSESSMENT OF FIBRE CONTENT AND 3D PROFILE IN CYLINDRICAL SFRC SPECIMENS
}

\author{
Sergio H. P. CAVALARO ${ }^{*}$, Rubén LÓPEZ-CARREÑO ${ }^{1}$, Josep María TORRENTS ${ }^{2}$, Antonio \\ AGUADO $^{1}$, Pablo JUAN-GARCÍA ${ }^{2}$ \\ ${ }^{1}$ Departamento de Ingeniería de la Construcción, ETSECCPB, Universidad Politécnica de Cataluña, BarcelonaTech, \\ Calle Jordi Girona 1-3, 08034, Barcelona, España \\ 2 Departamento de Ingeniería Electrónica, EEL, Universidad Politécnica de Cataluña, BarcelonaTech, Calle Jordi \\ Girona 1-3, 08034, Barcelona, España \\ * Corresponding author: Despacho 106, Edificio B1, Campus Nord, Departamento de Ingeniería de la Construcción, \\ ETSECCPB, Universidad Politécnica de Cataluña, BarcelonaTech, Calle Jordi Girona 1-3, 08034, Barcelona, España, \\ email sergio.pialarissi@upc.edu, Phone (+34) 934054247, Fax (+34) 934054135
}

\begin{abstract}
The inductive method proposed by Torrents et al. [1] and improved by Cavalaro et al. [2] is used to assess the fibre content and distribution in steel fibre reinforced concrete, providing valuable information for the design and the quality control. Despite several advantages, the method presents limitations. On one hand, it was conceived for the test of cubic specimens, which complicates its application in existing structures due to the difficulty to extract cubic cores. On the other hand, only a partial characterization of the fibre orientation is obtained given that the determination is restricted to the three axes of the specimen. With these measurements, it is not possible to derive the fibre orientation in other directions different from the ones used to test the sample. The objective of this paper is to propose an assessment of the fibre content and distribution in any direction using the inductive method and cylindrical specimens. For that, first a modification of the method is proposed. Then, new equations are deducted to generalize the test to samples with different shapes and to assess the anisotropy level as well as the directions with the maximum and the minimum fibre contribution. Next, an extensive experimental program and FEM numerical simulations are performed to validate and to determine the accuracy of the formulation developed. The results show that the application of these equations and the execution of only one additional measurement per specimen are enough to determine the fibre profile in all in-plane directions with a high accuracy.
\end{abstract}

Keywords: SFRC; inductive method; fibre content; orientation profile; quality control 


\section{INTRODUCTION}

The increasing use of steel fibre reinforced concrete (SFRC) [1-4] has generated the need for tests that provide information about the material. Besides the traditional tests to assess mechanical properties [5-7], different techniques are required to evaluate the fibre content and orientation. Both parameters are closely related with the quality and the performance of SFRC [3], being of interest for the design of structures and for the systematic quality control.

Several tests were developed with that purpose. An example is the inductive method proposed by Torrents et al. [1] and improved by Cavalaro et al. [2]. As shown in Figure 1.a, the equipment used in this test is composed by a LCR impedance analyser and a coil that receives an electric current and generates a magnetic field. When a SFRC cubic sample is placed inside the coil, a modification of the magnetic permeability of the medium is observed $[12,13]$. This modification leads to a change in the inductance [14] measured with the LCR impedance analyser. The method takes advantage of the fact that steel fibres are several orders of magnitude more magnetic than plain concrete [15]. This means that the test is highly sensitive to the inclusion of even small amounts of steel fibres, whereas the concrete practically do not affect the measurements regardless of its strength class or composition.
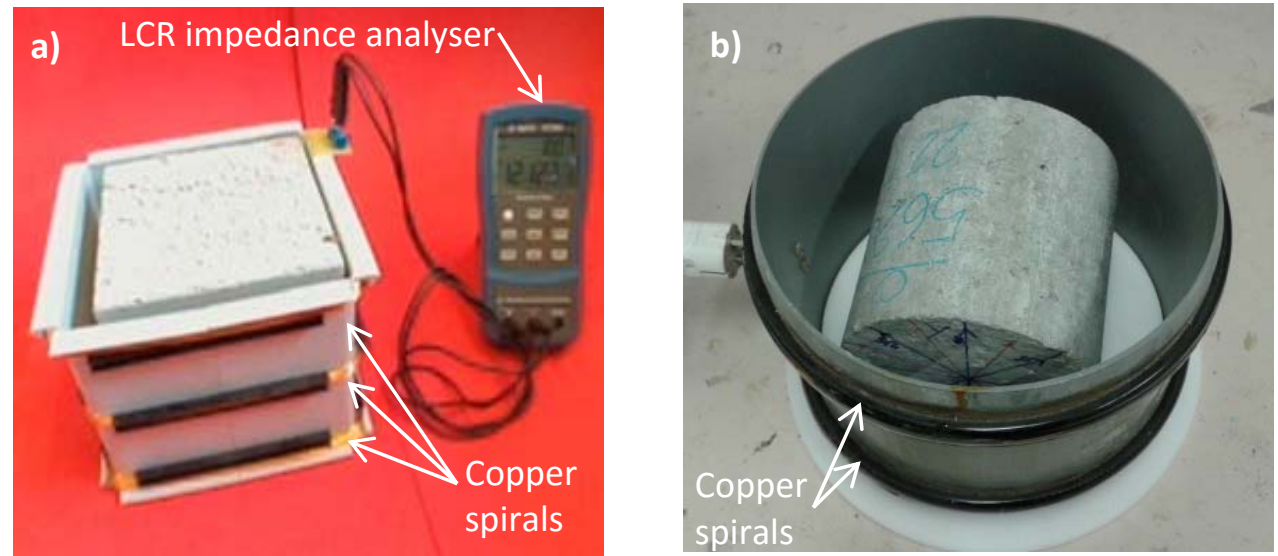

Figure 1. Inductive method by Torrents et al. [1] (a) and proposed modification (b)

The inductance change produced by the SFRC cubic specimen should be measured once in each main direction since the contribution of the fibres is proportional to the angle with the magnetic field inside the coil. Previous studies by Cavalaro et al. [2] proved that the summed inductance in the three axes is linearly related to the fibre content. The same authors also propose equations to estimate the orientation number based on the results obtained. This capability, together with the small cost of the equipment and the small time required for the characterization of each specimen, makes the inductive method an interesting alternative for the systematic quality control of SFRC [16-18].

Nevertheless, the method also presents limitations. For once, it was conceived and validated for the test of cubic SFRC specimens, which present symmetry in the three axes. In fact, the equations to predict the fibre content and orientation may not apply to specimens 
with other shapes that do exhibit such symmetry. This complicates the use of the method in existing structures (for instance segmental linings [19], pipes [20] or slabs [21]) due to the difficulty to obtain cubic extracted cores. In this context, the possibility of performing the test in cylindrical specimens would represent an improvement.

Furthermore, only a partial characterization of the fibre orientation is obtained with the method given that the determination is restricted to the three axes of the cubic specimen. Based on these results it is not possible to derive the orientation in different directions. In other words, the results provide no clear view on how the fibre distribution varies for directions different from the actually measured. If, for instance, the crack in the real structure should not coincide with these directions, it would not be possible to derive the orientation number and the fibre contribution perpendicular to the crack. This hinders the application of the test in elements such as slabs or shells in which the position of the failure plane is not well defined. It is also impossible to determine the degree of anisotropy of the sample or the directions with the highest and smallest contribution of the fibres.

The consideration of all these aspects related with the fibre distribution in real structures is one of the keystones of the most recent philosophy applied to the design of SFRC elements [23, 24], which account for the favourable or unfavourable orientations. In this context, the achievement of a more complete characterization of both moulded and extracted specimens is an essential step.

Taking that into account, the objective of this paper is to propose and validate the assessment of the fibre content and distribution in any direction using the inductive method and cylindrical specimens. For that, first a modification of the method is proposed. Then, new equations are deducted to generalize the test for samples with different shapes and to assess the fibre distribution. Next, an extensive experimental program and FEM numerical simulations are performed to validate and to determine the accuracy of the formulations proposed. The results obtained show that the application of these equations and the execution of only one additional measurement per specimen are enough to obtain the fibre distribution profile in any in-plane direction with a high accuracy. Now, parameters such as the minimum and maximum fibre orientation number, as well as the direction in which these values occur, may be easily obtained with the inductive method. This expands the potential of the technique, providing a reliable and simple tool to support the quality control and to supply the information required for a more refined design.

\section{MODIFICATION OF THE METHOD}

As in any method based in the inductance change, the accuracy of the measurement is highly dependent on the homogeneity of the magnetic field generated. For instance, imagine a field produced by a coil with a non-homogeneous flux distribution, as shown Figure 2.a. If the flux at one point $\left(\phi_{1}\right)$ has the double of the magnitude than at another point $\left(\phi_{2}\right)$, a fibre placed in $\phi_{1}$ will produce an inductance change twice as big as that placed in $\phi_{2}$. Therefore, the 
presence of non-homogeneities would cause the same inclusion to produce different response depending on the position inside the coil, thus compromising the reliability of the results.

a)

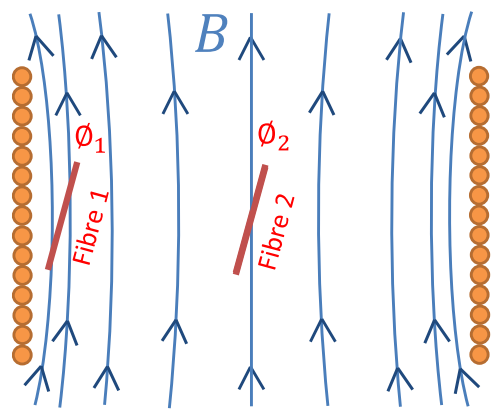

b)

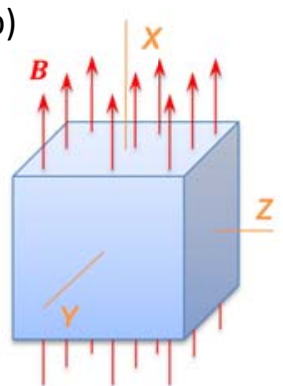

c)

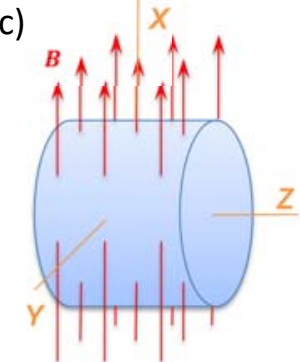

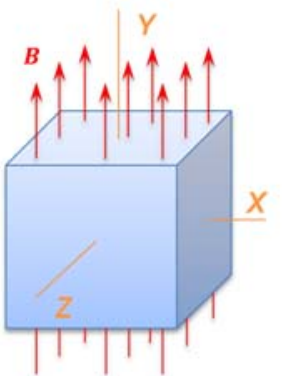
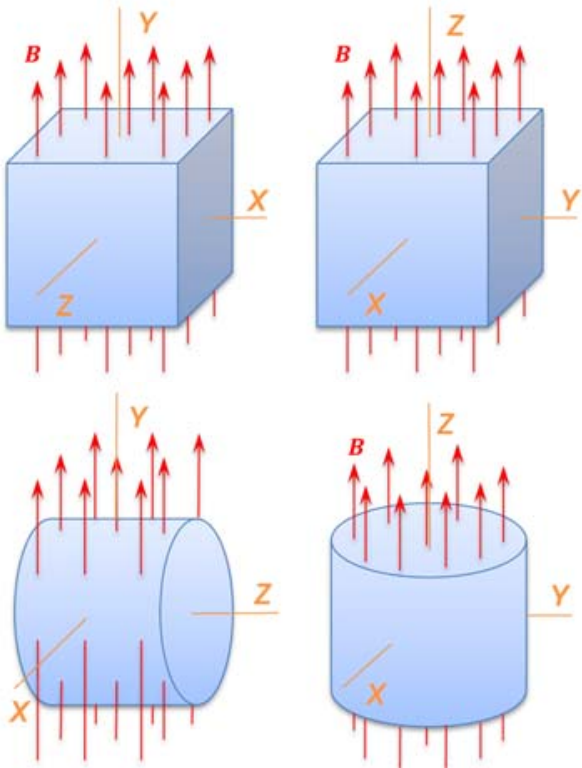

Figure 2. Fibres inside a non-homogeneous magnetic flux (a) and detail of magnetic field across cubic (b) and cylindrical (c) specimens

Two important inferences may be derived from this analogy. On one hand it becomes evident that to increase the accuracy of the method it is necessary to apply a magnetic field as uniform as possible. On the other hand, since a perfectly homogeny field may not be achieved in reality, the interaction between the magnetic field and the specimen will always depend on its position inside the coil.

The equipment proposed by Torrents et al. [1] is composed by a discontinuous square coil manufactured with a copper cable of $0.2 \mathrm{~mm}$ of diameter and a length of $1600 \mathrm{~mm}$, resulting in a total of 2354 turns. The dimensions of the prismatic plastic element around which the coil is placed are $15 \times 17 \times 17 \mathrm{~cm}$ (see Figure 1.a). It is known that the square is not the optimal cross section in terms of the homogeneity of the magnetic field generated inside the coil. In fact, the presence of corners contributes to variations in the field. Another associated problem arises if the test should be performed in specimens that do not show symmetry in the three axes, like in cylindrical cores. In this case, slight changes in the angle of the specimen relatively to the sides of the coil could induce additional scatter in the measurements. Both problems may be mitigated if a circular shaped coil would be used instead, leading to a more homogeny magnetic field and, ultimately, to less variability and more accurate results.

A different design of coil was proposed according with this idea in order to obtain a method suitable to cylindrical specimens. As shown in Figure 1.b, the new coil consisted of two spirals separated $13 \mathrm{~cm}$ apart and connected in a parallel discontinuous configuration. Each of them had a circular cross section with $25 \mathrm{~cm}$ of interior diameter and was made of a copper cable of $0.3 \mathrm{~mm}$ of diameter with a total of 1200 turns. 
In theory, the modification of the coil should not affect the equations proposed by Cavalaro et al. [2] to predict the fibre content and orientation number for cubic specimens. However, the deduction of more general equations that could be applied to the test of any shape of sample and to estimate the fibre distribution in any direction is still required.

\section{ANALYTICAL DEDUCTIONS}

\subsection{Fibre content}

In this study, equations were deducted to assess the fibre amount for a SFRC specimen with an unknown form and a volume $V$. To simplify the deduction, at first it is assumed that fibres are uniformly dispersed in the concrete mass. Regardless of its shape, the specimen could be discretised in the differential volumes $d V$ with the same fibre content ( $C_{f}$ in weight by unities of volume), as depicted in Figure 3.

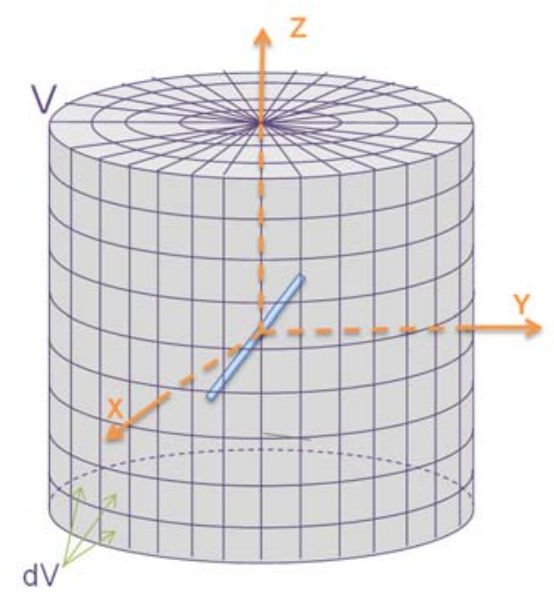

Figure 3. Discretization of a specimen

The inductance change $\left(d L_{i}\right)$ produced by each differential volume when measuring in an axis $i$ may be calculated according with Eq. 1, based on the study by Cavalaro et al [2]. This equation depends of the magnetic nature of the fibre $\left(k^{\prime}\right)$, the electric current $(I)$ that goes through the coil, the magnetic flux density $(B)$ generated in this point and the angel $\alpha_{i}$ formed between the magnetic field and the fibre in the differential element. The equation is also related with the shape factor $\gamma$ that is constant for each type of fibre and may be obtained by the ratio between the inductance of a single fibre perpendicular and parallel to the magnetic field.

$$
d L_{i}=\frac{k^{\prime} \cdot B \cdot C_{f}}{I} \cdot\left[\gamma+(1-\gamma) \cdot \cos ^{2} \alpha_{i}\right] \cdot d V=\frac{k^{\prime} \cdot B \cdot C_{f}}{I} \cdot \omega_{i} \cdot d V \quad \text { Eq. } 1
$$

Integrating Eq. 1 in the whole volume gives Eq. 2 for the global inductance change measured $L_{i}$ in the direction $i$. Notice that the parameter $B_{V, i}$ represents the integral of the 
magnetic field over the volume of the sample. Such parameter does not depend on the fibre used, being constant if the specimen is always placed in the same position and the coil is the same.

$$
L_{i}=\int_{V o l, i} d L_{i}=\frac{k^{\prime} \cdot \omega_{i} \cdot C_{f}}{I} \cdot \int_{V o l, i} B \cdot d V=\frac{k^{\prime} \cdot \omega_{i} \cdot C_{f}}{I} \cdot B_{V, i} \quad E q .2
$$

The sum of the inductance changes produced after measuring in three orthogonal axes $(x, y$ and $z$ indicated in Figure 5$)$ yields Eq. 3 . It is easy to demonstrate that in cubic specimens, the values of $B_{V, x}, B_{V, y}$ and $B_{V, z}$ are equal due to the symmetry of the sample. Consequently, Eq. 3 may be reduced to Eq. 4 . The latter could be further simplified since the angles $\alpha_{x}, \alpha_{y}$ and $\alpha_{z}$ are complementary, meaning that $\omega_{x}+\omega_{y}+\omega_{z}$ should equal the constant $1+2 \cdot \gamma$. Notice that all parameters in Eq. 4 are constant except for the fibre content and the summed inductance, which should hold a linear relation with each other. Consequently, if the constant of proportionality $\left(\beta^{\prime}\right)$ is known in advance, the content of fibres could be estimated based on the summed inductance obtained in the test. This agrees with the demonstrated by Cavalaro et al. [2] and could be represented according with Eq. 5

$$
\begin{array}{cc}
\sum_{x, y, z} L_{i}=\frac{k^{\prime} \cdot C_{f}}{I} \cdot\left(\omega_{x} \cdot B_{V, x}+\omega_{y} \cdot B_{V, y}+\omega_{z} \cdot B_{V, z}\right) & \text { Eq. } 3 \\
\sum_{x, y, z} L_{c u b, i}=\frac{k^{\prime} \cdot C_{f}}{I} \cdot B_{V, x} \cdot\left(\omega_{x}+\omega_{y}+\omega_{z}\right)=\frac{k^{\prime} \cdot C_{f}}{I} \cdot B_{V, x} \cdot(1+2 \cdot \gamma) & \text { Eq. } 4 \\
C_{f}=\beta^{\prime} \sum_{x, y, z} L_{c u b, i} & \text { Eq. } 5
\end{array}
$$

However, if a specimen without symmetry was tested, the values of $B_{V, x}, B_{V, y}$ and $B_{V, z}$ would not be the same. As a result, the simplifications used to obtain Eq. 4 and 5 would not apply, meaning that the relation between the summed inductance and the fibre content should not be linear. Consequently, it would not be possible to assess the fibre content through the inductive method. To eliminate this problem and make the method applicable to any shape of specimen, a mathematical artifice was used. Instead of calculating the summed inductance in the three axes, the sum of the ratio between the measurement in each axis and the corresponding constant $B_{V, i}$ should be used according with Eq. 6 .

$$
L_{e}=\sum_{x, y, z} \frac{L_{i}}{B_{V, i}}=\frac{k^{\prime} \cdot C_{f}}{I} \cdot\left(\omega_{x}+\omega_{y}+\omega_{z}\right)=\frac{k^{\prime} \cdot C_{f}}{I} \cdot(1+2 \cdot \gamma)
$$

Eq. 6 
Notice that the latter will reduce every time to $\omega_{x}+\omega_{y}+\omega_{z}$, which is constant and equal to $1+2 \cdot \gamma$. In other words, the summed equivalent inductance $\left(L_{e}\right)$ should always be linearly related with the fibre content. This suggests that the content of fibre could be estimated with $L_{e}$ through Eq. 7 regardless of the shape of the specimen, being the formulation proposed by Cavalaro et al. [2] and Torrents et al. [1] only a special case of it. In fact, Eq. 7 becomes Eq. 5 if cubic specimens are considered.

$$
C_{f}=\beta \sum_{x, y, z} \frac{L_{i}}{B_{V, i}}=\beta \cdot L_{e}
$$

Comparing Eq. 6 and 7, it is possible to deduct the proportionality constant $\beta$ (see Eq. 8). It is usual to determine $\beta$ with one or two specimens by dividing the fibre content weighted after crushing the specimen and the corresponding value of equivalent inductance $\left(L_{e}\right)$ measured. This division gives the slope of the straight line that passes through the origin of the coordinate system and relates the measurements of the inductive method and the fibre content. Alternatively, this parameter may be assessed directly by using a known content of fibres that is subjected to the inductive method in the three directions. Notice that according with the new formulation proposed, $\beta$ should be the same for any shape of specimen and concrete type.

$$
\beta=\frac{I}{k^{\prime} \cdot(1+2 \cdot \gamma)}
$$

Eq. 8

\subsection{Orientation number and contribution of fibres}

For the deduction of the equations that determine the fibre distribution consider the same example from section 3.1. To simplify the initial deduction assume that all fibres are arranged with the same direction. The orientation number $\left(\eta_{i}\right)$ is given by the average of the cosine of the angle formed between the fibres and a line parallel to at an axis $i$ [25-27]. The average of the cosine - equivalent to the average orientation number in the direction $i$ - may be obtained by isolating the $\cos \alpha_{i}$ in Eq.2. This gives Eq. 9, which may be combined with Eq. 7 and 8 to derive Eq. 10 for the assessment of the orientation number of a general specimen with all fibres arranges in the same direction. It is important to remark that Eq. 10 reduces to the proposed by Cavalaro et al. [2] if cubic specimens are considered.

$$
\begin{gathered}
\eta_{i}=\cos \alpha_{i}=\sqrt{\frac{1}{1-\gamma} \cdot\left(L_{i} \cdot \frac{I}{k^{\prime} \cdot C_{f} \cdot B_{V, i}}-\gamma\right)} \\
\eta_{i}=\sqrt{\frac{L_{i} \cdot(1+2 \cdot \gamma)-L_{e} \cdot B_{V, i} \cdot \gamma}{L_{e} \cdot B_{V, i} \cdot(1-\gamma)}}
\end{gathered}
$$


Eq. 10 is representative of a situation in which all fibres are aligned in the same direction. In this context, the average of the cosine of the angle with the axis $i$ equals the square root of the average square cosine. Consequently, the orientation number may be easily estimated through the results from the inductive method. Nevertheless, if the fibres are dispersed with certain randomness, an overestimation could be obtained with Eq. 10 since the average cosine of the angle with the axis $i$ diverges from square root of the average square cosine obtained with the inductive method. This mathematical incongruence was previously explained by Cavalaro et al [2] who deducted that the average overestimation ( $\mu$ ) per axis may range from 0.07 and 0.10 depending on the specimen shape.

In order to compensate for this overestimation, the correction parameter $\mu$ must be introduced in Eq. 10. Another parameter $(\vartheta)$ should be included to account for the nonhomogeneity of the magnetic field that is not considered in the mathematical deductions. As a result of both modifications, Eq. 11 is obtained for the prediction of the orientation number in a general specimen with dispersed fibres. By definition, the fibre contribution $\left(C_{i}\right)$ is still calculated through Eq. 12.

$$
\begin{gathered}
\eta_{i}=\vartheta \cdot \sqrt{\frac{L_{i} \cdot(1+2 \cdot \gamma)-L_{e} \cdot B_{V, i} \cdot \gamma}{L_{e} \cdot B_{V, i} \cdot(1-\gamma)}}-\mu \\
C_{i}=\frac{\eta_{i}}{\sum_{i=x, y, z} \eta_{i}}
\end{gathered}
$$

\subsection{Generalization for cylindrical specimens}

The equations derived for the assessment of the content of fibres (Eq. 7) and the orientation numbers (Eq. 11) are valid for any type of specimen. To apply them, it is only necessary to assess the constants $\mathrm{B}_{\mathrm{V}, \mathrm{i}}, \mu$ and $\vartheta$ corresponding to the shape of specimen and the coil used. These parameters are not easy to obtain analytically since the assessment of the magnetic field at any point requires solving a complex set of equations. A more direct approach considered in this study consists of the application of an electromagnetic finite element. The mesh used is composed by cubic brick elements with a side of $1 \mathrm{~mm}$. The magnetic flux density (B) and the magnetic field (H) follow Eq. 13 and Eq. 14, with J being the electric current density. These are the classic equations from electromagnetism theory that are valid for static magnetic fields, in which the wavelength produced by the current is much bigger than the dimensions of specimens or of the coil.

$$
\nabla \cdot \vec{B}=0
$$




$$
\nabla \times \vec{H}=J
$$

Once the magnetic field is calculated it may be integrated over the volume occupied by the specimen to obtain $\mathrm{B}_{\mathrm{V}, \mathrm{i}}$. The estimation of $\mu$ and $\vartheta$ requires the consideration in the finite element of the concrete and the dispersed fibre inside the coil. For that, the algorithm to simulate the fibre distribution and the analogy for the inductance change proposed by Cavalaro et al. [2] are taken into account. It is important to remark that this model has been validated with experimental results.

Each SFRC specimen is simulated with the magnetic field acting in the three main directions, thus reproducing the procedure conducted during the test. After performing several simulations, the parameters $\mu$ and $\vartheta$ are estimated. Table 1 summarizes the parameters for the assessment of the fibre content and orientation in any cylindrical and cubic specimens. Notice that these parameters remain constant regardless of the concrete mix, fibre content and type.

Table 1. Constant parameters for cylindrical and cubic specimens

\begin{tabular}{|c|c|c|c|c|c|c|}
\hline \multirow{2}{*}{ Shape } & \multirow{2}{*}{ Size (mm) } & \multicolumn{5}{|c|}{ Parameter } \\
\cline { 3 - 7 } & & $\mathrm{B}_{\mathrm{V}, \mathrm{x}}$ & $\mathrm{B}_{\mathrm{V}, \mathrm{y}}$ & $\mathrm{B}_{\mathrm{V}, \mathrm{z}}$ & $\mu$ & $\vartheta$ \\
\hline \multirow{2}{*}{ Cylindrical } & $\phi 100 \times 100$ & 536 & 536 & 538 & 0.085 & 1.03 \\
\cline { 2 - 7 } & $\phi 150 \times 150$ & 1789 & 1789 & 1809 & 0.085 & 1.03 \\
\hline \multirow{2}{*}{ Cubic } & $100 \times 100 \times 100$ & 695 & 695 & 695 & 0.100 & 1.03 \\
\cline { 2 - 7 } & $150 \times 150 \times 150$ & 2342 & 2342 & 2342 & 0.100 & 1.03 \\
\hline
\end{tabular}

\subsection{Orientation profile for cylindrical specimens}

The use of cylindrical specimens not only is feasible through the general equations proposed here, but also opens up the possibility of overcoming one of the main disadvantage of the inductive method in its current configuration. As mentioned before, the method is not capable of providing the fibre orientation in axes different from the ones used to measure the inductance. Therefore, limited information is obtained when the crack does not coincide with these directions and it is not possible to assess the anisotropy of the material in terms of the maximum and minimum orientation number and their corresponding directions.

In any SFRC cylindrical specimen, the fibre distribution may be understood as the superposition of two samples: one isotropic (Figure 4.a) and one with certain anisotropy (Figure 4.b). If the inductance measurements are taken by spinning both samples at several angles $(\theta)$, different behaviours would be observed. As shown in the graph from Figure 4.a, the isotropic sample would show a constant inductance $\left(L_{\text {iso }}\right)$ since an evenly distributed number of fibres is present in all directions. On the contrary, as depicted in the graph from Figure 4.b, the inductance of the anisotropic sample should present maximum and minimums values that coincide with the directions with more and less fibre contribution, respectively. Notice that the 
minimum value could never be equal to 0 since the fibre produce an inductance change even if placed perpendicular to the magnetic field. In this context, the final inductance measured in the real specimen should be the result of the sum of the curves obtained for the isotropic and anisotropic specimens (see Figure 4.c).

a)
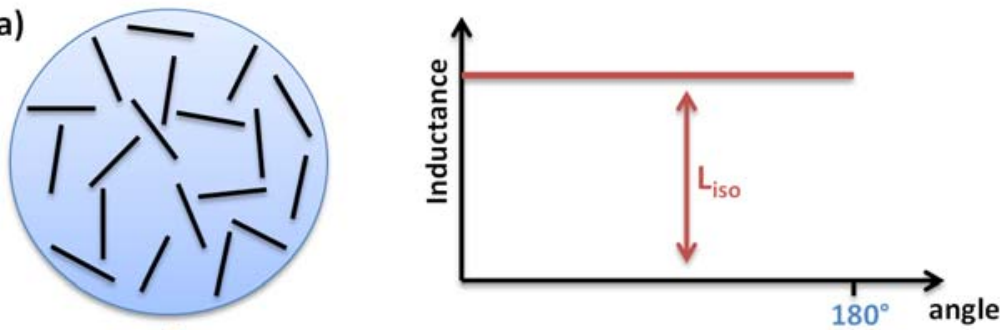

b)
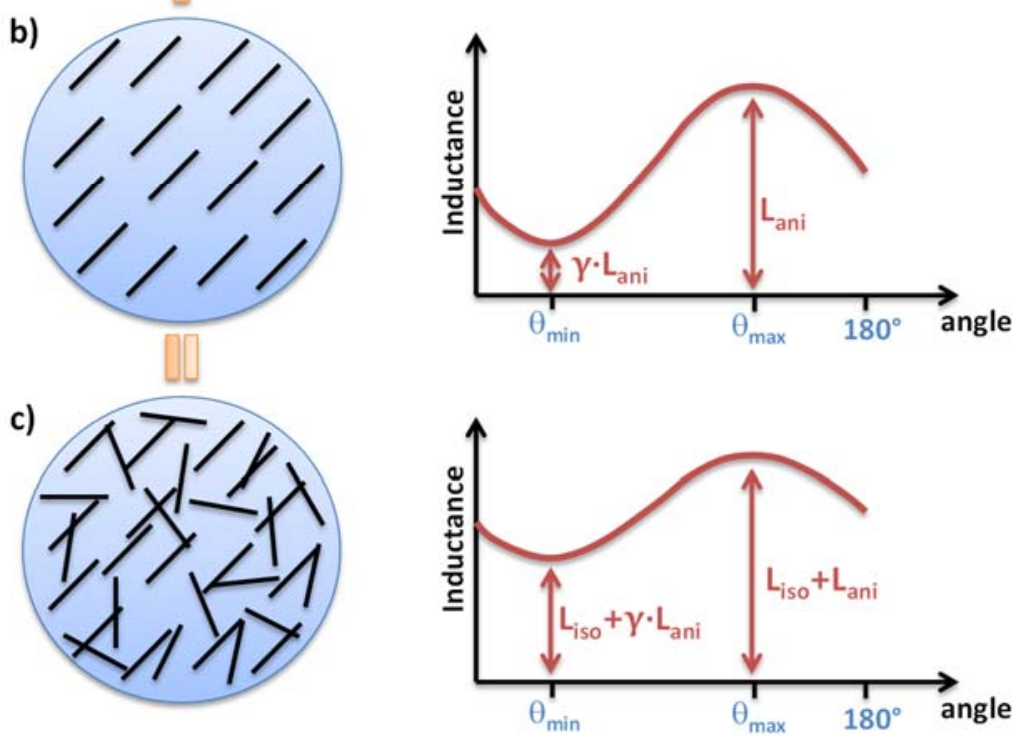

Figure 4. Detail and inductance profile of isotropic (a), anisotropic (b) and resultant (c) specimens

Results by Laranjeira [25] and Grunewald [27] suggest that the variation of the angle formed by the fibre and a certain axis may be represented through a Gauss or a Gumble distribution. This indicates that the curve obtained for the anisotropic sample tend to have a continuous shape with a clear maximum and minimum values. Consequently, it would be possible to use the equations proposed in this study to predict the variation of the orientation number depending $\theta$ without the need of performing several additional measurements.

Making an analogy with the equations deducted in section 3.2, the inductive change $\left(\mathrm{L}_{\theta}\right)$ at a certain angle $\theta$ could be estimated mathematically through Eq. 15 that reflects the superposition of two parts. The first of them represents the isotropic sample that should yield a constant inductance equal to $L_{\text {iso. }}$. The second part represents the variation of the anisotropic sample that may be approximated by means of Eq. 2, considering a maximum inductance Lani $_{\text {i }}$ observed at the angle with maximum fibre contribution $\left(\theta_{\max }\right)$. 


$$
L_{\theta}=L_{\text {iso }}+L_{\text {ani }} \cdot\left[\gamma+(1-\gamma) \cdot \cos ^{2}\left(\theta+\theta_{\max }\right)\right]
$$

Notice that the only unknowns that should be determined to obtain the change in inductance are $L_{\text {iso, }} L_{\text {ani }}$ and $\theta_{\max }$, which will be constant for each specimen. To obtain them, a system of three equations must be solved. Therefore, three measurements of inductance in the $X Y$ plane are required. Given that by default one measurement is already taken in the $X$ axis and another in the $Y$ axis, it is possible to derive the complete profile of fibre distribution with only one additional assessment in the $X Y$ plane. In order to improve the reliability of the predictions, the additional measurement should be performed in the intermediate direction that forms and $45^{\circ}$ angle with $X$ and $Y$, as depicted in Figure 5 . It is important to remark that this represents a change in the usual procedure applied up to date in the inductive method. Instead of performing 3 measurements, 4 are required to achieve a complete characterization of the specimen. By convention, it is assumed that the axes $X$ and $Y$ coincide with the angle of $0^{\circ}$ and $90^{\circ}$, respectively.

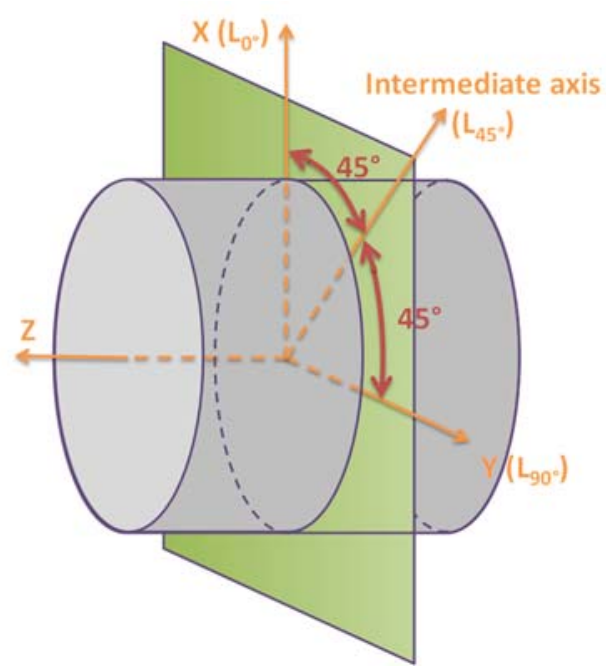

Figure 5. Measurement axes to obtain the orientation profile

Solving the system of equations for the measurements in $X\left(L_{0^{\circ}}\right), Y\left(L_{90^{\circ}}\right)$ and the intermediate direction $\left(L_{45^{\circ}}\right)$ gives Eq. 16,17 and 18 for the estimation of $L_{a n i}$, $L_{\text {iso }}$ and $\theta_{\max }$. Using Eq. 15 , it is also possible to obtain the angle that will give the minimum inductance $\left(\theta_{\min }\right)$ through Eq. 19. Notice that the formulation for the assessment of $\theta_{\max }$ has an initial sign that might be positive or negative since two angles satisfy the arc cosine. A simple mathematical proof should be performed with equation Eq. 15 to determine the correct angle. The results obtained for the angles of $0^{\circ}, 45^{\circ}$ and $90^{\circ}$ are compared with the measured experimentally that served as input parameters for the analysis. The correct angle should provide identical results for all measurements.

$$
L_{\text {ani }}=\frac{\sqrt{2}}{1-\gamma} \sqrt{\left(L_{0^{\circ}}-L_{90^{\circ}}\right)^{2}+\left(L_{0^{\circ}}+L_{90^{\circ}}-2 \cdot L_{45^{\circ}}\right)^{2}}
$$




$$
\begin{gathered}
L_{i s o}=\frac{L_{0^{\circ}}+L_{90^{\circ}}-L_{a n i} \cdot(1+\gamma)}{2} \\
\theta_{\max }= \pm \frac{1}{2} \cdot \operatorname{acos}\left[\frac{L_{0^{\circ}}-L_{90^{\circ}}}{L_{a n i} \cdot(1-\gamma)}\right] \\
\theta_{\min }=\theta_{\max }-90^{\circ}
\end{gathered}
$$

Eq. 18

Eq. 19

The orientation number may now be estimated at any axis with an angle $\theta$ by introducing in Eq. 12 the inductances $\mathrm{L}_{\theta}$ and $\mathrm{L}_{\theta+90^{\circ}}$ estimated with Eq.15 for the same angle and for $\theta+90^{\circ}$. This is represented mathematically through Eq. 20 .

$$
\eta_{\theta}=1.03 \cdot \sqrt{\frac{L_{\theta} \cdot(1+\gamma)-\left(L_{\theta+90^{\circ}}+L_{z} \cdot \frac{B_{V, x}}{B_{V, z}}\right) \cdot \gamma}{\left(L_{\theta}+L_{\theta+90^{\circ}}+L_{z} \cdot \frac{B_{V, x}}{B_{V, z}}\right) \cdot(1-\gamma)}}-0.085
$$

\subsection{Isotropy of the fibre distribution}

The maximum $\left(L_{\max }\right)$ and the minimum $\left(L_{\min }\right)$ inductance may be assessed by introducing Eq. 16 and 17 in Eq. 15. The results are presented in Eq. 21 and 22.

$$
\begin{array}{cc}
L_{\text {max }}=L_{\text {iso }}+L_{\text {ani }} & \text { Eq. } 21 \\
L_{\text {min }}=L_{\text {iso }}+\gamma \cdot L_{\text {ani }} & \text { Eq. } 22
\end{array}
$$

As indicated by Eq. 19, the maximum and the minimum value of inductance should be separated by $90^{\circ}$. Consequently, the maximum $\left(\eta_{\max }\right)$ and the minimum $\left(\eta_{\min }\right)$ orientation numbers corresponding to the same angles may be estimated by using $L_{\max }$ and $L_{\min }$ in Eq. 20. This leads to Eq. 23 and 24, respectively.

$$
\eta_{\max }=1.03 \cdot \sqrt{\frac{L_{\max } \cdot(1+\gamma)-\left(L_{\min }+L_{z} \cdot \frac{B_{V, x}}{B_{V, z}}\right) \cdot \gamma}{\left(L_{\max }+L_{\min }+L_{z} \cdot \frac{B_{V, x}}{B_{V, z}}\right) \cdot(1-\gamma)}}-0.085
$$




$$
\eta_{\min }=1.03 \cdot \sqrt[2]{\frac{L_{\min } \cdot(1+\gamma)-\left(L_{\max }+L_{z} \cdot \frac{B_{V, x}}{B_{V, z}}\right) \cdot \gamma}{\left(L_{\max }+L_{\min }+L_{z} \cdot \frac{B_{V, x}}{B_{V, z}}\right) \cdot(1-\gamma)}}-0.085 \quad \text { Eq. } 24
$$

The possibility of estimating the complete orientation profile with one additional inductance measurement allows the proposal of a parameter related with the level of isotropy of SFRC. This parameter, called isotropy factor $(\Omega)$, is defined in Eq. 25 as the ratio between $\eta_{\min }$ and $\eta_{\max }$. It may assume values ranging from nearly 0 to 1 . If the fibre distribution is perfectly isotropic, $\eta_{\max }$ and $\eta_{\min }$ acquire the same value and $\Omega$ equals 1 . Otherwise, if all the fibres are aligned in the same direction, the value of $\eta_{\max }$ is orders of magnitude bigger than $\eta_{\min }$ and the $\Omega$ tends to 0 .

$$
\Omega=\frac{\eta_{\min }}{\eta_{\max }}
$$

\section{EXPERIMENTAL VALIDATION}

In total, three experimental programs were conducted in order to confirm the accuracy of the equations deducted in the present study. One of them was dedicated to validate the formulations proposed for assessing the fibre content in section 3.1. Another was performed to contrast the equations for assessing the orientation number and the contribution of the fibres from sections 3.2 and 3.3. A third experimental program was conducted to confirm the predictions for the orientation profile based on the formulation proposed in sections 3.4 and 3.5.

\subsection{Fibre content}

\subsubsection{Materials and methods}

In order to evaluate the fibre content, cubic specimens with $150 \mathrm{~mm}$ of edge and cylindrical specimens with $150 \mathrm{~mm}$ of diameter and height were tested with the inductive method and then crushed to assess the weight of fibre. The specimens were cast with 6 concrete mixes, using 2 types of concretes (conventional and self-compacting) as well as 3 nominal fibre contents $\left(30,45\right.$ and $\left.60 \mathrm{~kg} / \mathrm{m}^{3}\right)$.

The steel fibres used in the SFRC were BASF Masterfiber 502 with a circular crosssection of $1 \mathrm{~mm}$ diameter, $50 \mathrm{~mm}$ of length and hooked ends. These fibres are made of low carbon steel with approximately 3000 unities per $\mathrm{kg}$. A 250 litres vertical mixer was used to produce batches of $120 \mathrm{I}$, which was enough to cast all samples of the same mix. Table 1 presents the composition of the concrete mixes tested and their fresh state properties 
measured. For each mix and geometry, 4 specimens were produced in accordance with EN 12390-2.

Table 2. Concrete mixes tested

\begin{tabular}{|c|c|c|c|c|c|c|c|}
\hline \multirow{2}{*}{ Components } & \multirow{2}{*}{ Characteristics } & \multicolumn{6}{|c|}{ Content $\left(\mathrm{kg} / \mathrm{m}^{3}\right)$} \\
\hline & & \multicolumn{3}{|c|}{ Conventional } & \multicolumn{3}{|c|}{ Self-compacting } \\
\hline Gravel $(12 / 20 \mathrm{~mm})$ & Granite & \multicolumn{3}{|c|}{810} & \multicolumn{3}{|c|}{200} \\
\hline Gravel $(5 / 12 \mathrm{~mm})$ & Granite & \multicolumn{3}{|c|}{404} & \multicolumn{3}{|c|}{500} \\
\hline Sand $(0 / 5 \mathrm{~mm})$ & Granite & \multicolumn{3}{|c|}{817} & \multicolumn{3}{|c|}{1200} \\
\hline Cement & CEM I 52,5 R & \multicolumn{3}{|c|}{312} & \multicolumn{3}{|c|}{380} \\
\hline Water & - & \multicolumn{3}{|c|}{156} & \multicolumn{3}{|c|}{165} \\
\hline Superplasticizer & Glenium TC 1425 & \multicolumn{3}{|c|}{2.19} & \multicolumn{3}{|c|}{4.56} \\
\hline Hidratation activator & X SEED & \multicolumn{3}{|c|}{6.24} & \multicolumn{3}{|c|}{7.6} \\
\hline Fibres & Steel fibres & 30 & 45 & 60 & 30 & 45 & 60 \\
\hline \multicolumn{2}{|c|}{ Reference } & CC30 & CC45 & CC60 & SC30 & SC45 & SC60 \\
\hline \multicolumn{2}{|c|}{ Slump (mm) according UNE 83503} & 3 & 5 & 3 & - & - & - \\
\hline \multicolumn{2}{|c|}{ Flow extent $(\mathrm{mm})$ according EN 206} & - & - & - & 650 & 650 & 670 \\
\hline
\end{tabular}

In the first step of the testing procedure, the three main axes of the specimen were marked. In the case of cylindrical samples, the $Z$ axis was the revolution axis parallel to the casting direction, whereas the axes $X$ and $Y$ were randomly selected. For the cubic, the $Z$ axis was parallel to the casting direction while the axes $X$ and $Y$ were parallel to the sides of the moulds. The second step consisted of measuring the inductance for the three directions. For that, the modified coil shown in Figure 1.b was used. The input of the electrical current and the inductance measurements were performed with the equipment AGILENT LCR 4263B. The electrical current was set as alternating with a frequency of $1 \mathrm{kHz}$ and a voltage of $1 \mathrm{~V}$.

Finally, the fibre content was estimated according with the EN 14721. A hydraulic press was used to crack the specimens that were then crushed in a grinding machine. Afterwards, the fibres were manually separated with the help of a magnet and weighted. Considering the laborious and long time required, this procedure was performed only in 20 specimens: 10 cylindrical and 10 cubic. In each group, 5 specimens were of conventional concrete and 5 were of self-compacting concrete.

In this study, the accuracy of the formulation developed is evaluated in terms of the trueness and the precision. The trueness is given by the mean difference between the real values measured in the experimental program and the estimated with the equations developed in previous sections. It marks how close the average predictions are from the reality, being small values indicative of high accuracy. The precision is obtained as the the standard deviation of the differences between estimated and real values. It provides information on how individual values might vary around the average. Once more, small values indicate higher precision.

\subsubsection{Results and analysis}

Figure 6 shows the fibre content $\left(C_{f}\right)$ assessed after crushing and the equivalent inductance $\left(L_{e}\right)$ calculated from the inductances as proposed in section 3.1 for cubic and 
cylindrical specimens. It is evident that a linear relation exists between $L_{e}$ and $C_{f}$. Notice that despite considering specimens of different shapes, the results fit to the same linear regression with a $\mathrm{R}^{2}$ of 0.99 . The linear regression starts approximately at the origin of the coordinate system and assumes a slope $\beta$ approximately equal to 5909.4 .

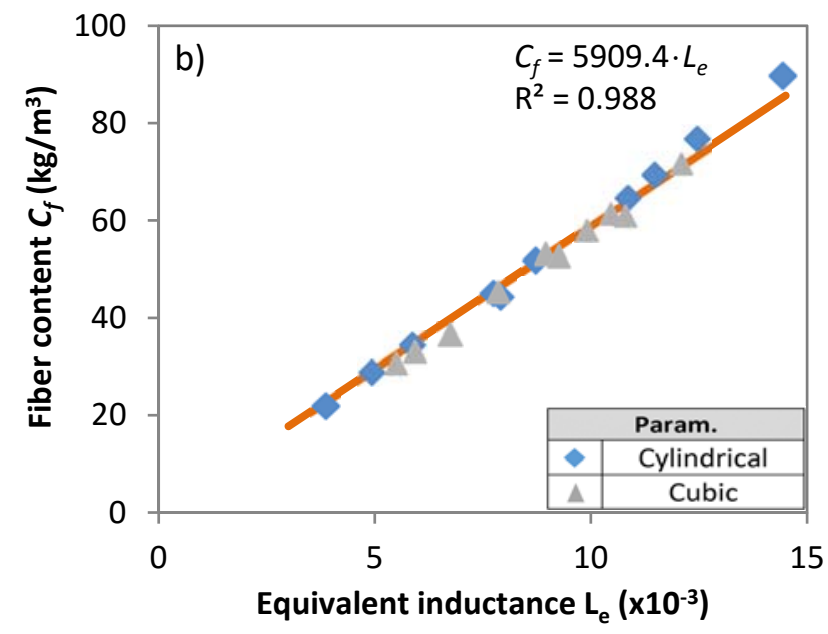

Figure 6. Relation between equivalent Inductance and fibre content

The average trueness calculated as de difference between the fibre content measured and the predicted with the equivalent inductance is $0.38 \mathrm{~kg} / \mathrm{m}^{3}$. The value obtained considering only the cylindrical or the cubic specimens are $0.46 \mathrm{~kg} / \mathrm{m}^{3}$ and $-0.33 \mathrm{~kg} / \mathrm{m}^{3}$, respectively. Such small errors may be considered negligible taking into account the usual fibre content in SFRC and the wide range measured in the experimental program (from $20 \mathrm{~kg} / \mathrm{m}^{3}$ to almost $100 \mathrm{~kg} / \mathrm{m}^{3}$ ), thus confirming the high accuracy of the method. These results validate the formulation proposed in section 3.1 to estimate the fibre content. It confirms that the consideration of the summed equivalent inductance $\left(L_{e}\right)$ should be the reference parameter to assess the fibre content since the same calibration curve applies regardless of the shape of specimen characterized. Likewise, good predictions are achieved for both conventional and self-compacting concrete.

\subsection{Orientation of fibres}

\subsubsection{Materials and methods}

With the intent of evaluating the equations for the orientation number and the contribution of fibres, a cylindrical specimen with a known fibre orientation was made by hand. For that, 13 layers of non-magnetic cardboard sheets were cut in a circular shape and glued together in order to form a specimen with $150 \mathrm{~mm}$ of diameter and $150 \mathrm{~mm}$ of height, as shown in Figure 7. 


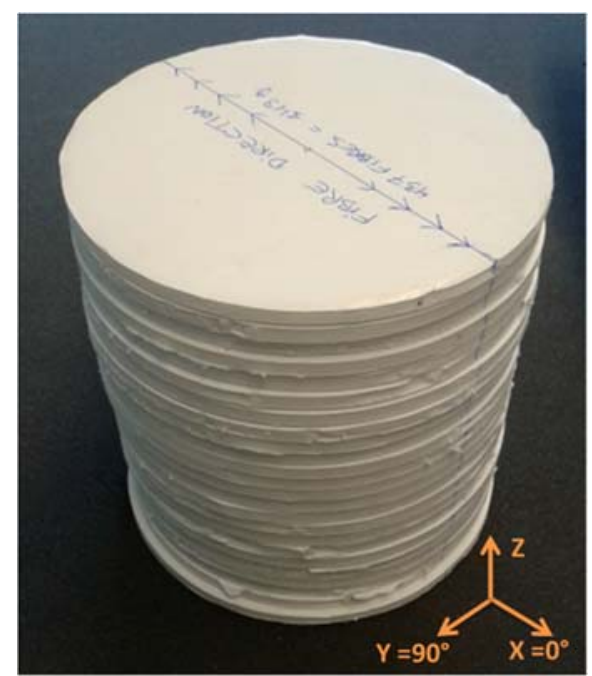

Figure 7. Cardboard specimen with aligned fibres

In total, $243 \mathrm{~g}$ of fibres of the same type used in the first experimental program were placed between adjacent sheets. All fibres were distributed uniformly along the height with the same alignment, being orthogonal to the theoretical axis of the specimen ( $Z$ axis). The specimen was then tested with the inductive method. Measurements were taken at the $Z$ axis and in the $X Y$ plane for the angles of $0^{\circ}, 10^{\circ}, 20^{\circ}, 30^{\circ}, 40^{\circ}, 50^{\circ}, 60^{\circ}, 70^{\circ}, 80^{\circ}$ and $90^{\circ}$ between the alignment of the fibres and the direction of the magnetic field.

\subsubsection{Results and analysis}

Figure 8.a shows the curves for the real orientation number and the estimated with Eq. 10 by using the results of the inductive method. Both curves practically coincide. The trueness and the precision from the proposal indicate an error of prediction that might be considered negligible, again confirming the accuracy of the equations developed here.
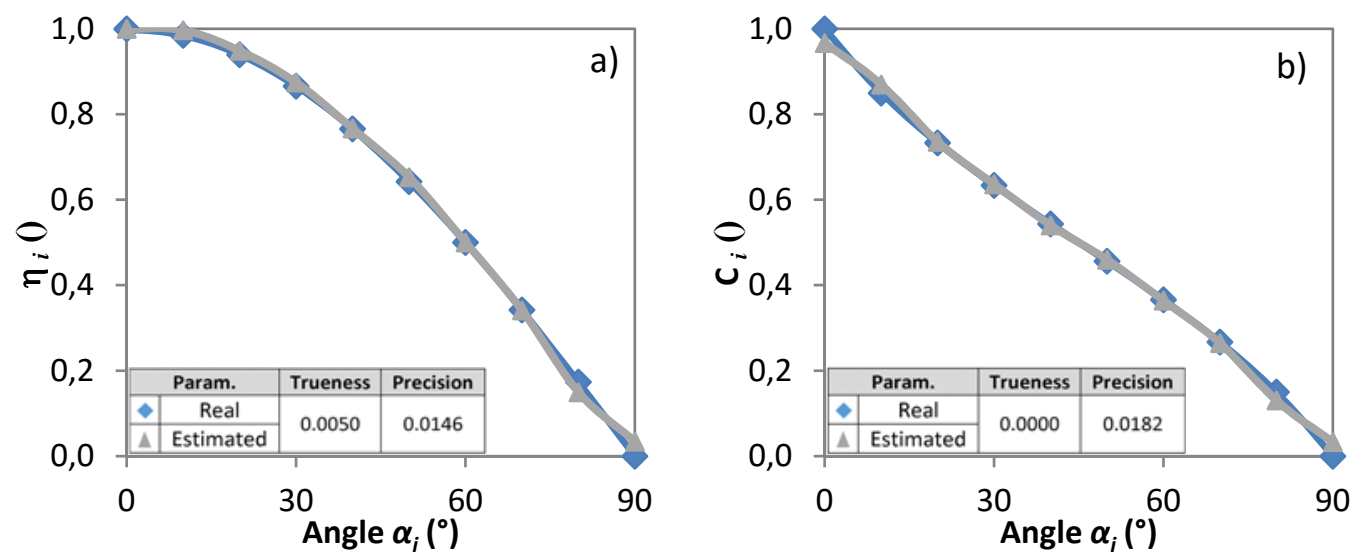

Figure 8. Variation of the orientation number (a) and the fibre contribution (b) depending on the angle of measurement for the cardboard specimen 
Figure 8.b presents the real contribution of fibres and the estimated with the values of inductance and Eq. 12. In the same way as the orientation number, the real and the calculated curves practically overlap. Once more, the low values of trueness and precision suggest a negligible error in the predictions. The high accuracy corroborates the efficacy of the equations and confirms that the integrals of magnetic flux density were well calculated.

\subsection{Orientation profile}

\subsubsection{Materials and methods}

The 24 cylindrical specimens produced in the first experimental program were also used to validate the equations proposed to obtain the orientation profile. The aim was to evaluate if the predictions with Eq. 15 to 19 agrees with the values actually measured. For that, the specimens were marked with 8 directions of measurement in the XY plane separated $22.5^{\circ}$ from each other, as shown in Figure 9. The inductance was evaluated in each direction as well as along the $Z$ axis. To avoid favouring a better fit of the data, the directions used to estimate the orientation profile were selected randomly prior to the test.

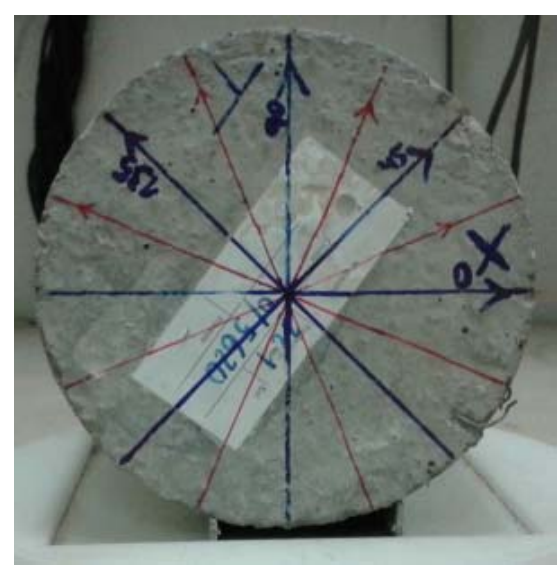

Figure 9. Axes of measurements in a cylindrical specimen

\subsubsection{Results and analysis}

Figure 10.a shows the orientation profile measured and estimated for two of the specimen characterized. The estimations were performed with Eq. 15 to 18 and the inductances for the angles of $0^{\circ}, 45^{\circ}$ and $90^{\circ}$. All samples presented a similar trend regardless of the fibre content or the concrete type. The results reveal that the complete inductance profile is well reproduced by Eq. 15 to 18 . As predicted, the profiles show the same period (180 degrees) but different amplitudes that is related with the level of anisotropy of the samples. 

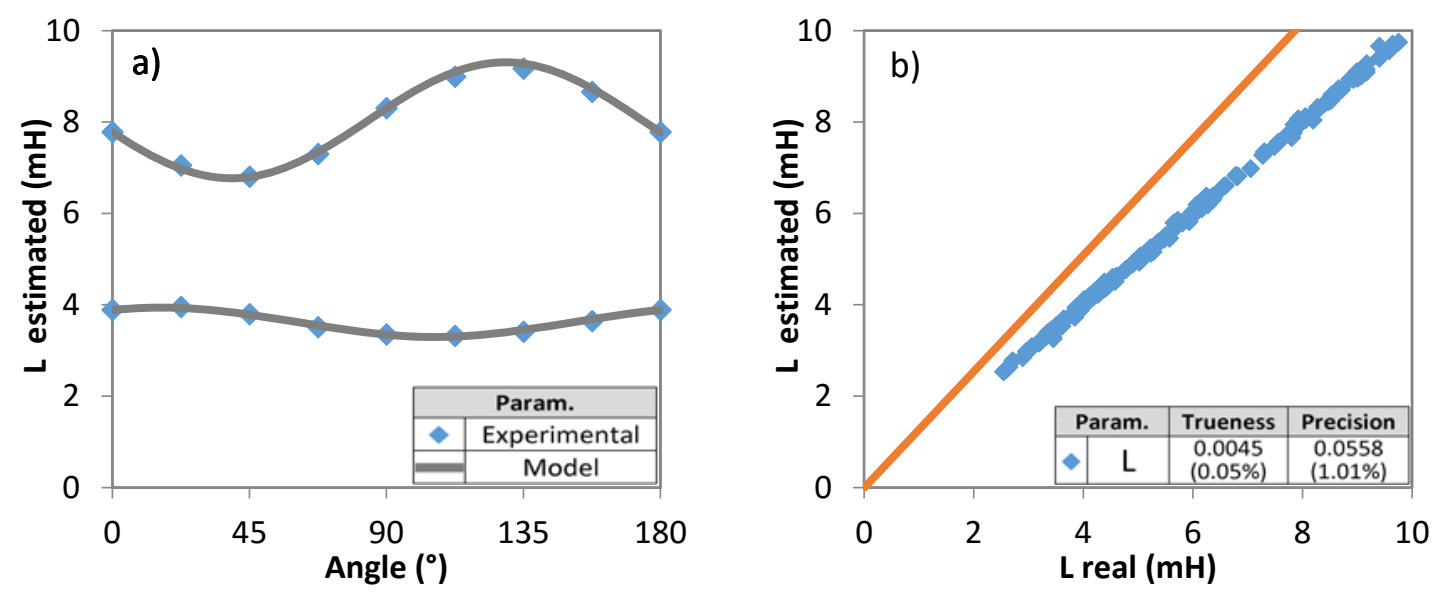

Figure 10. Inductance profile (a) and comparison between real and estimated values (b)

Figure 10.b presents the real inductance measured at different angles and the estimated with Eq. 15 to 19 for all samples tested. It is evident that the equations proposed are capable of predicting the experimental results with a high accuracy. In fact, the theoretical estimation had a trueness of $0.04 \%$ and a precision of $1.01 \%$ in relation with the measurements. Both results confirm that it is feasible to estimate the inductance and the orientation profile with only one additional measurement in the XY plane.

Figure 11 presents the comparison between the real values obtained in the experimental program and the ones estimated with the simplified equations deducted previously. This evaluation is performed for the maximum $\left(L_{\max }\right)$ and the minimum $\left(L_{\min }\right)$ inductance calculated, as well as for the direction $\left(\theta_{\max }\right)$ with the maximum contribution and the direction $\left(\theta_{\min }\right)$ with the minimum contribution. In the case of the experimental results, a linear regression was used to assess these parameters, whereas in the analytical approach the equations proposed here were used with the measurements at $0^{\circ}, 45^{\circ}$ and $90^{\circ}$.
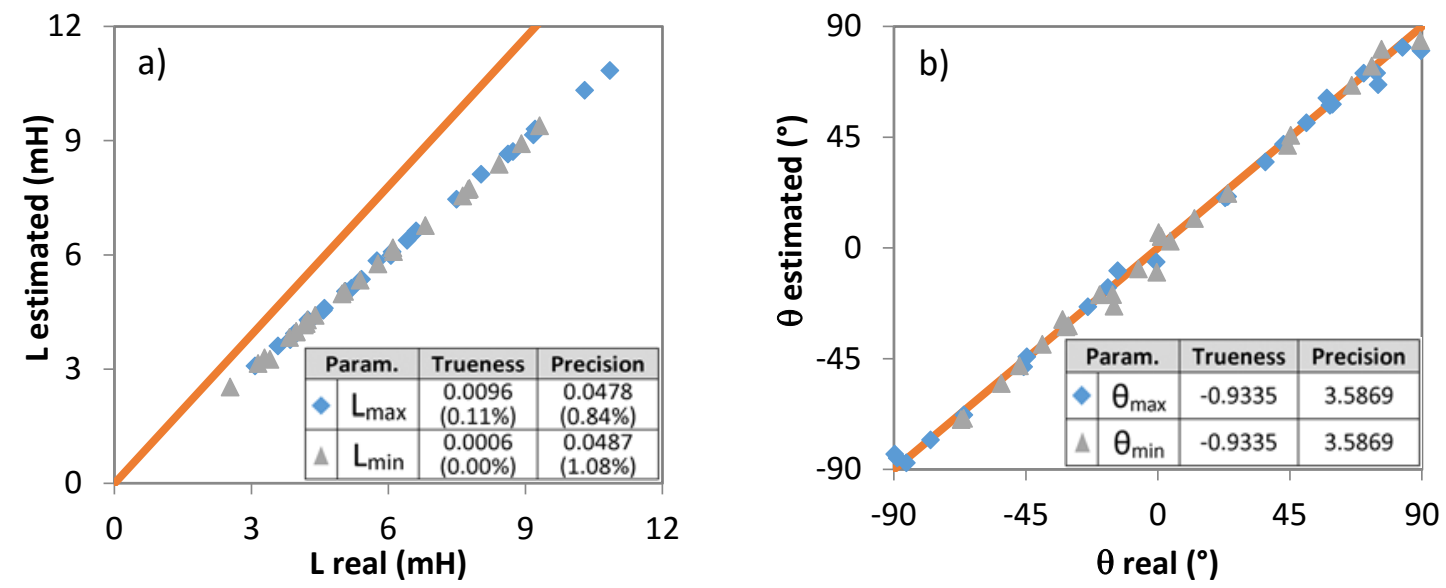

Figure 11. Comparison between real and estimated values for $L_{\max }(a), L_{\max }(b), \theta_{\max }(c)$ and for $\theta_{\min }(d)$ based on the experimental results 
The figures reveal that the basic parameters that determine the fibre distribution profile are well predicted by the model developed. A small error is obtained in all cases. Observe, for instance, the error of prediction of the direction with the maximum and the minimum fibre contribution. In both cases, the real values are predicted with a trueness smaller than $1^{\circ}$. Similar outcome is also verified for the maximum and the minimum inductance, in which even better predictions are achieved.

\section{NUMERICAL VALIDATION}

The experimental programs demonstrate the effectiveness of the formulation proposed here. Although specimens with perfectly aligned fibres or with 3 nominal fibre contents and 2 concrete types were tested, it is important to consider that in practice other levels of anisotropy may occur. Moreover, although only cast samples were characterized, in practice it should be also feasible to obtain the fibre distribution for extracted cores. In this context, it is advisable to evaluate the equations proposed for cast and extracted samples with a wider range of anisotropy levels. However, this is not easy to perform experimentally provided the difficulty to control de fibre distribution and the limitation in terms of resources to assess such a wide range of concrete types.

Therefore, instead of performing this verification based on additional experimental results, a numerical approach similar to the already conducted by Cavalaro et al. [2] was performed. The viability of this approach was confirmed by the authors for $15 \mathrm{~cm}$ edge cubic specimens and for a square coil. In the present study, a similar finite element model (FEM) was first developed and validated. Then it was used to evaluate the accuracy of the formulation proposed for the assessment of the orientation number, the contribution and the orientation profile.

\subsection{Description and validation of the FEM}

The FEM developed contained two modules. In the first of them, several probabilistic laws are responsible for defining the distribution and the orientation of each fibre within a concrete specimen that could be either cast in a mould or extracted from an existing structure. Aspects such as the geometry of the sample, the wall-effect of the formwork in cast specimens [28-30] and the cut of fibres in extracted specimens were taken into account. Once the fibres are distributed inside the specimen, the second module calculates the inductance change produced in the circular coil when the inductive test is performed. The magnetic field generated by the coil is implemented simulating the same conditions as in the experimental program. Finally, with the inductance change and the distribution of fibres, the model is able to compare the real fibre distribution and the predicted with the inductance measurements. For more details on the FEM, check Cavalaro et al [2]. 
To validate the FEM applied in the numerical study, the cardboard specimen from the experimental program described in section 4.2 was simulated and the inductance changes were assessed for several angles. Figure 12 presents the inductances estimated with the FEM and measured in the laboratory for equivalent angles. The values of trueness and precision of the inductance predicted with the FEM are 0.0735 and 0.1632 , respectively. Such values indicate that the FEM is capable of reproducing the inductance change in the coil due to the presence of fibres.

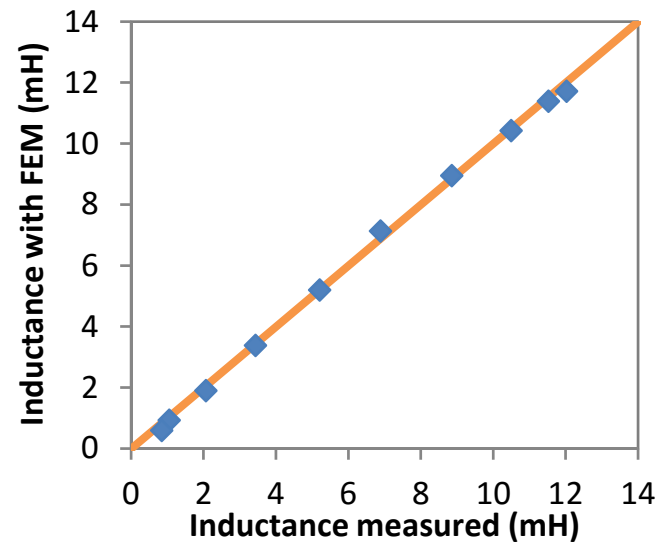

Figure 12. Comparison between the inductance measured in the laboratory and the results of the FEM for a cylindrical coil

\subsection{Parametric study}

Once the FEM was validated simulations were performed considering a wide range of levels of anisotropy in the fibre distribution. The different distributions were achieved by modifying the values of the director vectors $V_{D t_{i, \min }}$ and $V_{D t_{i, \max }}$ that govern the probability of finding fibres in each of the main axes. For instance, if all axes shared the same value of these parameters, an approximately isotropic distribution would be obtained in zones not influence by the wall-effect. Consequently, almost the same number of fibres would be observed in all directions. On the contrary, if one axis presented a smaller $V_{D t_{i, \min }}$ or $V_{D t_{i, \max }}$, fibres would have a lower probability of appearing alongside directions that approach this axis. In the simulations $V_{D t_{i, \text { min }}}$ varied from -0.5 to -1.0 and $V_{D t_{i, \max }}$ ranged from 0.5 to 1.0 .

Each level of anisotropy was simulated for $\gamma$ of $0.000,0.025$ and 0.050 that account for the inclusion of fibres with different aspect ratios. A shape factor of 0 represents a theoretical situation in which the diameter of the fibre is negligible in comparison with its length. On the other hand, a shape factor of 0.05 is representative of the fibre used in the experimental program.

The combination of parameters led to 63 cases, all of them consisting of the simulation of the inductive test of cylindrical cast specimens with fibre content of $60 \mathrm{~kg} / \mathrm{m}^{3}$. For each case, 20 models were analysed in order to derive a representative sample. This approach is necessary since the probabilistic laws behind the fibre distribution in the FEM produce a slightly different specimen every time. Therefore, a minimum number of models are required 
to perceive a clear trend. Specimens were simulated and the inductance change was assessed in the $Z$ axis and at every $15^{\circ}$ in the $X Y$ plane.

These simulations were repeated for extracted cores. Contrarily to the observed in cast specimens that have a fibre orientation induced by the lateral surface of the moulds, the extracted cores are not affected by this wall-effect. Instead it presents several fibres that are cut during the extraction process. To simulate these conditions, no lateral restriction to the position of the fibre was considered. Furthermore, the stretches of fibre that extend beyond the lateral extraction boundary were eliminated since in reality they would be cut.

\subsection{Results and analysis}

\subsubsection{Orientation number}

Figure 13.a shows the relation between the real orientation number of the specimens modelled with the FEM and the calculated with Eq. 11 using the inductance change for the same specimens. The results from the simulations performed with cast and extracted samples are included in the figure. Notice that the orientation numbers obtained cover the usual range found in practice, reaching even extreme values that unlikely would be observed. Despite that, the estimated orientation numbers agree with the real ones in the whole range considered. In fact, the trueness and the precision of the estimations suggest an average error far below $0.5 \%$.
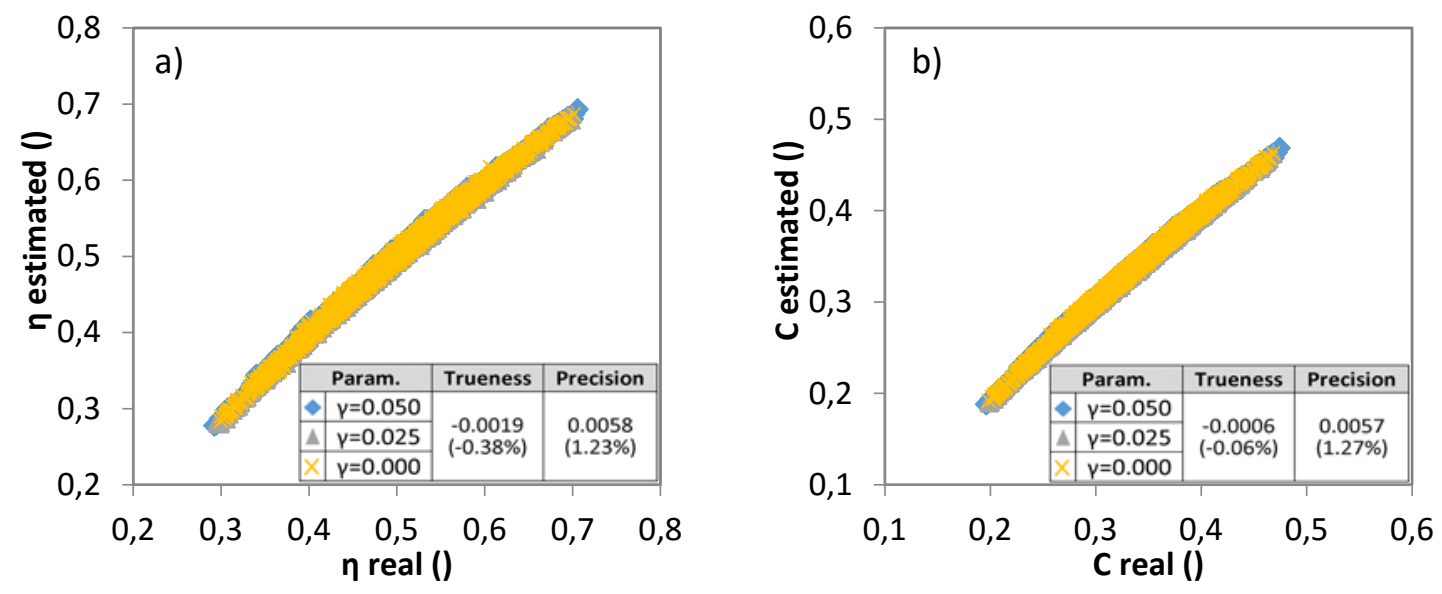

Figure 13. Comparison between real and estimated orientation number (a) and fibre contribution (b) for cast and extracted samples

Figure 13.b shows analogous results for the fibre contribution using Eq. 12. Such results confirm the accuracy of Eq. 11 and 12 for the assessment of fibre contribution and orientation number based on the inductive method applied for cylindrical specimens. This remains true regardless of the type of sample, the aspect ratio of the fibres or the degree of anisotropy. 


\subsubsection{Orientation profile}

The inductance obtained with the FEM at the angles of $0^{\circ}, 45^{\circ}$ and $90^{\circ}$ in the $\mathrm{XY}$ plane were used to derive the complete inductance profile according with Eq. 15 to 19 for any direction. This profile is then compared with the real ones. This comparison for all models considered in the parametric study is presented in Figures 14.a.
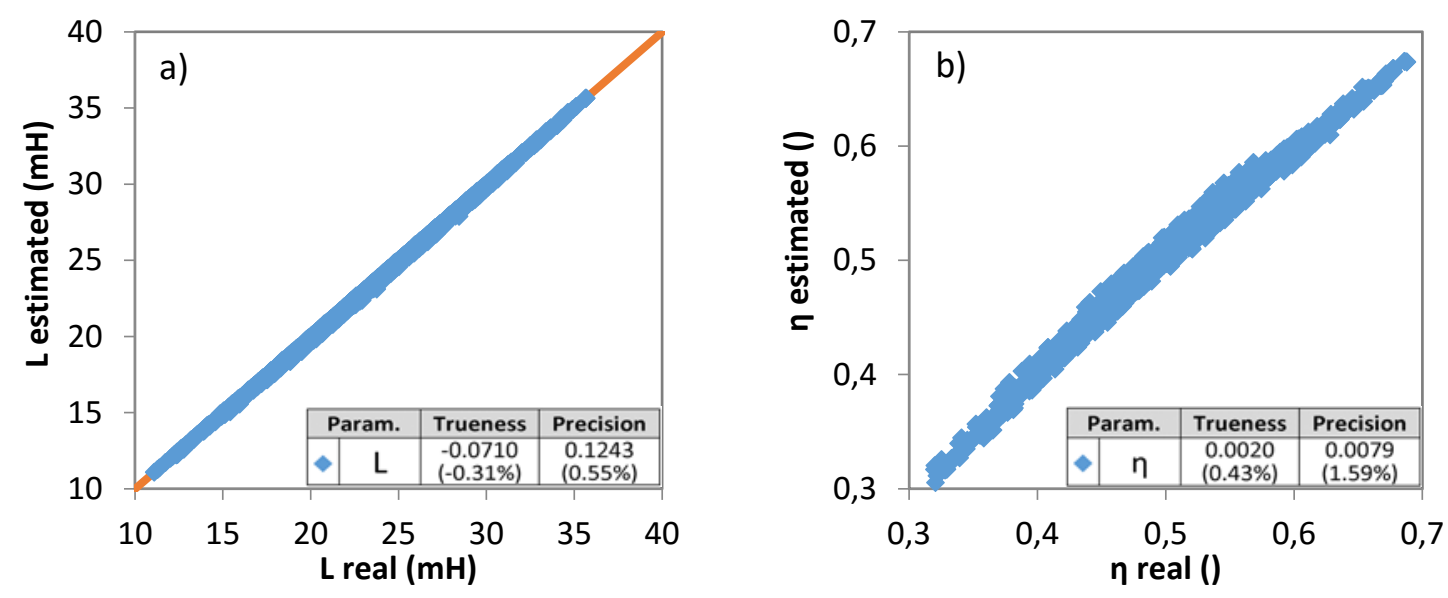

Figure 14. Comparison between real and estimated inductance profile (a) and orientation profile (b)

It is observed that the profile calculated with the FEM agrees with the obtained by using the simplified formulation developed here. The same outcome remains true when orientation profiles estimated with Eq. 20 are compared with the actual fibre distribution from the specimen, as shown in Figure 14.b. This confirms that the estimation of the inductance and the orientation profile is possible with only one additional measurement for a wide range of levels of anisotropy and different fibre types. In fact, the new formulation proposed and the test of cylindrical specimens allows detecting the orientation number in axes different from the ones used for the measurement, thus providing a much clearer picture of the fibre distribution in SFRC elements.

Verifications were also performed to evaluate the accuracy of the predictions of $L_{\max }$, $L_{\min }, \theta_{\max }, \theta_{\min }, \eta_{\max }, \eta_{\min }$, and $\Omega$ according with Eq. 21, 22, 18, 19, 23, 24 and 25, respectively. The estimations were compared with the values calculated for the same specimens with the FEM. Figure 15 presents the results obtained. 

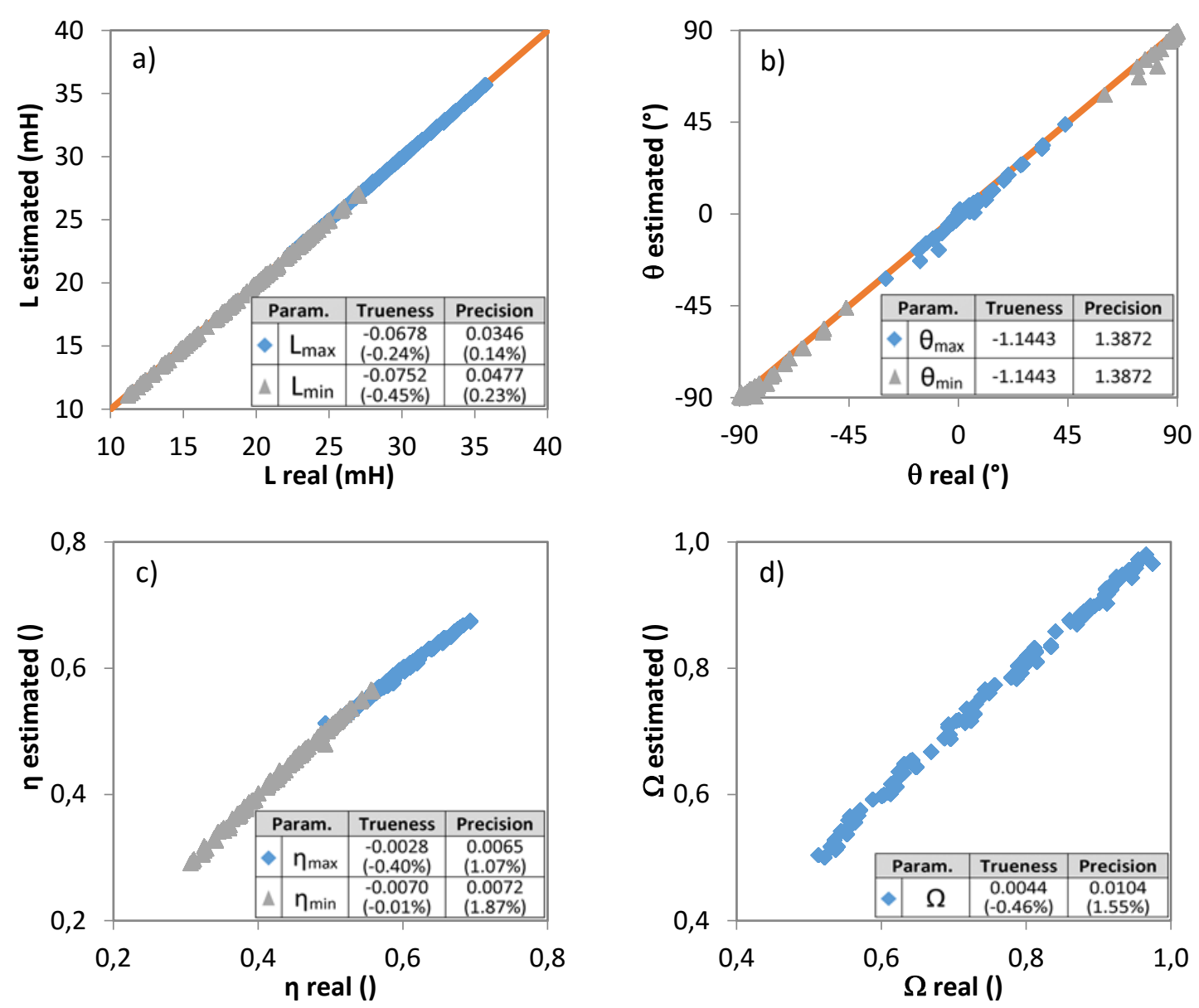

Figure 15. Comparison between real and estimated values for $L_{\max }$ and $L_{\min }(a), \theta_{\max }$ and $\theta_{\min }(b)$, $\eta_{\max }$ and $\eta_{\text {mix }}(c)$ and $\Omega(d)$ based on the numerical analysis

In all cases, the formulation proposed remains accurate even if extreme values are considered. This is observed especially for the maximum and minimum inductance and orientation number, which show average errors below $0.5 \%$. Good results are also obtained for $\theta_{\max }$ and $\theta_{\min }$, with a trueness that indicates an average error of prediction below $1.5^{\circ}$. In the comparison shown in Figure 15.d between the real and the estimated isotropy factor $(\Omega)$ a noteworthy fit is obtained regardless of the level of isotropy because of the accurate prediction of $\eta_{\max }$ and $\eta_{\min }$.

\section{CONCLUSIONS}

In this work, an increase in the capability of predicting the fibre content and distribution with the inductive method applied to specimens with any shape was achieved. After analytical deductions, several equations were proposed and then validated with an extensive experimental and numerical study. The following are the main conclusions derived from this work. 
- A modified coil was designed. This coil is more compatible with the test of specimens that do not present symmetry in the three axes (such as the cylindrical ones) since it tends to reduce potential variability in the results.

- The analytical deductions indicate that the formulation currently used to predict the fibre content in cubic specimens could lead to errors if applied to specimens without symmetry in the three axes. A new approach valid regardless of the shape of the specimen and based on the equivalent inductance $\left(L_{e}\right)$ was proposed in the present study. The experimental program performed with cubic and cylindrical specimens confirm that with this approach the same calibration curve applies to both specimens with an average error of only $380 \mathrm{~g} / \mathrm{m}^{3}$. The good accuracy is verified for both conventional and self-compacting concrete.

- The numerical validation shows that the formulation proposed to determine the orientation number of the fibres (Eq. 11) and their contribution in one direction (Eq. 12) based on the results from the inductive method in cylindrical specimens is capable of estimating the real values with an average trueness below $0.5 \%$. Negligible errors of prediction are obtained in cast or extracted specimens for a wide range of orientation numbers.

- The equations deducted expand the application of the inductive method, allowing the determination of the orientation number in any direction (Eq. 15 and 20). The validity of these equations was confirmed experimentally and in numerical simulations. A high accuracy of the predictions is obtained in both cases, with trueness and precision values that are below $0.43 \%$ and $1.69 \%$, respectively.

- The inductive method may now be used to assess in a simplified way the maximum and minimum orientation numbers (Eq. 23 and 24), as well as the directions in which these values occur (Eq. 18 and 19). Such assessment may serve to determine potential planes of weakness of real scale element. Moreover, a new parameter was proposed to quantify the degree of anisotropy of SFRC (Eq. 25) based on the maximum and the minimum orientation number. The experimental and the numerical validations indicate that all these parameter may be obtained only by applying the equations proposed here and by performing 4 measurements per sample instead of the 3 currently used. This minute additional effort leads to a much more complete assessment of the characteristics of SFRC, providing the information required for the quality control and the design according with the most recent guidelines.

\section{ACKNOWLEDGEMENTS}

The authors thank PROMSA for the support during the experimental program and the Ministerio de Economía y Competitividad for the financial support provided within the project FIBHAC (IPT-2011-1613-420000). 


\section{REFERENCES}

[1] Torrents JM, Blanco A, Pujadas P, Aguado A, Juan-García P, Sánchez-Moragues MÁ (2012) Inductive method for assessing the amount and orientation of steel fibers in concrete. Mater Struct 45(10):1577-1592

[2] Cavalaro SHP, López R, Torrents JM, Aguado A (2014) Improved assessment of fibre content and orientation with inductive method in SFRC. Mater Struct, 1-15

[3] Serna P, Arango S, Ribeiro T, Núñez AM, Garcia-Taengua E (2009) Structural cast-inplace SFRC: technology, control criteria and recent applications in Spain. Mater Struct 42(9):1233-1246

[4] di Prisco M, Plizzari G, Vandewalle L (2009) Fibre reinforced concrete: new design perspectives. Mater Struct 42(9):1261-1281

[5] CEN. EN 14651:2005 (2005) Test method for metallic fibrered concrete - Measuring the flexural tensile strength (limit of proportionality (LOP), residual), European Committee for Standardization, Brussels

[6] RILEM TC 162-TDF (2003) Test and design methods for steel fibre reinforced concrete$\sigma-\varepsilon$ design method: final recommendation. Mater Struct 36(262):560-567

[7] IBN. NBN B 15-238 (1992) Essais des bétons renforcés de fibres - Essai de flexion sur éprouvettes prismatiques, Institut Belge de Normalisation Brussels (In French)

[8] Mobasher B, Stang H, Shah SP (1990) Microcracking in fiber reinforced concrete. Cem Concr Res 20(5):665-676

[9] Pujadas P, Blanco A, Cavalaro SHP, de la Fuente A, Aguado A (2014) Multidirectional double punch test to assess the post-cracking behaviour and fibre orientation of FRC. Constr Build Mater 58:214-224

[10] Ferrara L, Meda A (2006) Relationships between fibre distribution, workability and the mechanical properties of SFRC applied to precast roof elements. Mater Struct 39(4):411-420

[11] Blanco A (2013) Characterization and modelling of SFRC elements. PhD Thesis, Universitat Politècnica de Catalunya

[12] Polder D, Van Santeen JH (1946) The effective permeability of mixtures of solids. Physica 12(5):257-271.

[13] Sihvola AH, Lindell IV (1992) Effective permeability of mixtures. Progr Electromagn Res 6:153-180 
[14] Faifer M, Ferrara L, Ottoboni R, Toscani S (2013) Low Frequency Electrical and Magnetic Methods for Non-Destructive Analysis of Fiber Dispersion in Fiber Reinforced Cementitious Composites: An Overview. Sensors 13(1):1300-1318

[15] Ferrara L, Faifer M, Toscani S (2012) A magnetic method for non destructive monitoring of fiber dispersion and orientation in steel fiber reinforced cementitious composites-part 1: method calibration. Mater Struct 45(4):575-589

[16] Maturana A, Sanchez R, Canales J, Orbe A, Ansola R, Veguería E (2010) Technical economic analysis of steel fibre reinforced concrete flag slabs. A real building application. XXXVII IAHSWorld Congress on Housing

[17] Orbe A, Cuadrado J, Losada R, Rojí E (2012) Framework for the design and analysis of steel fiber reinforced self-compacting concrete structures. Constr Build Mater 35:676686

[18] Orbe A, Rojí E, Losada R, Cuadrado J (2014) Calibration patterns for predicting residual strengths of steel fibre reinforced concrete (SFRC). Compos Part B-Eng 58:408-417

[19] de la Fuente A, Pujadas P, Blanco A, Aguado A (2011) Experiences in Barcelona with the use of fibres in segmental linings. Tunn Undergr Space Technol 27(1):60-71

[20] de la Fuente A, Escariz RC, de Figueiredo AD, Molins C, Aguado A (2012). A new design method for steel fibre reinforced concrete pipes. Constr Build Mater 30:547-555

[21] Pujadas P, Blanco A, de la Fuente A, Aguado A (2013) Cracking behavior of FRC slabs with traditional reinforcement. Mater Struct 45(10):1577-1592

[22] Pujadas P, Blanco A, Cavalaro SHP, Aguado A (2014) Plastic fibres as the only reinforcement for flat suspended slabs: experimental investigation and numerical simulation. Constr Build Mater 57:92-104

[23] Laranjeira de Oliveira F (2010) Design-oriented constitutive model for steel fiber reinforced concrete. PhD Thesis, Universitat Politècnica de Catalunya

[24] CEB-FIB (2010) Model Code. Comité Euro-International du Beton-Federation International de la Precontraint, Paris

[25] Laranjeira F, Grünewald S, Walraven J, Blom C, Molins C, Aguado A (2011) Characterization of the orientation profile of steel fiber reinforced concrete. Mater Struct 44(6):1093-1111

[26] Laranjeira F, Aguado A, Molins C, Grünewald S, Walraven J, Cavalaro S (2012) Framework to predict the orientation of fibers in FRC: a novel philosophy. Cem Concr Res 42(6):752-768

[27] Grünewald S (2004) Performance-based design of selfcompacting fibre reinforced concrete. PhD Thesis, Delft University of Technology 
[28] Kameswara Rao CVS (1979) Effectiveness of random fibres in composites. Cem Concr Res 9(6):685-693

[29] Soroushian P, Lee CD (1990) Distribution and orientation of fibers in steel fiber reinforced concrete. ACI Mater J 87(5):433-439

[30] Martinie L, Roussel N (2011) Simple tools for fiber orientation prediction in industrial practice. Cem Concr Res 41(10):993-1000 